\title{
Modified shouldice repair of bilateral inguinal hernia with hydrocele: A case report
}

\author{
Smit Shah ${ }^{l}$, Dr. Praful Shah ${ }^{2}$
}

${ }^{1}$ MS-3, Rutgers Robert Wood Johnson Medical School, NJ, USA. ${ }^{2}$ M.S. General Surgeon, Fellow of Minimal Access Surgery,

\begin{abstract}
Indirect inguinal hernia is a common medical condition that can be caused by an embryological defect due to failure of closure of processus vaginalis that can lead to herniation (i.e. abnormal protrusion) of abdominal contents into scrotal sac in males. In this paper, we describe an interesting case report of a patient with bilateral inguinal hernia with hydroceles who underwent a modified shouldice repair. In addition, we also discuss initial presentation \& surgical management of this patient who had painless postero-inferior displacement of testes. Reason why we think this case is unique, is because of 'bilateral' hydroceles along with bilateral inguinal hernia which is rarely seen as compared to its unilateral counterparts. We also describe various surgical steps of modified shouldice repair with bilateral orchiopexy along with the post-operative outcome. Finally, we also discuss various types of hydroceles, along with its pathology that is found after surgical resection.
\end{abstract}

Keywords: Transilluminable, mass, modified, shouldice, painless.

\section{Introduction}

Indirect inguinal hernia is a common medical condition that is caused by an embryological defect due to failure of closure of processus vaginalis that can lead to herniation (i.e. abnormal protrusion) of abdominal contents into scrotal sac in males. The most common location of indirect inguinal hernia is lateral to inferior epigastric artery. Due to a defect in the deep inguinal ring, patients most commonly present with an inguinal mass. However, embryological defects can vary and cause atypical presentation of indirect inguinal hernia along with hydrocele. Hydrocele is a fluid filled serous cavity that is found most commonly adjacent to the affected testicle and is caused by failure of processus vaginalis to close proximally and distally along with decreased fluid reabsorption in the affected spermatic cord. In contrast, direct inguinal hernia occurs secondary to defect in the superficial inguinal ring, which leads to herniation of bowel tissue medial to inferior epigastric artery. Direct inguinal hernias most commonly occur due to weakness in the fascia of the abdominal wall. Finally, femoral hernias lie inferior to inguinal ligament and are located medial to femoral vein. "Approximately 96 percent of groin hernias are inguinal and 4 percent are femoral". Major risk factors include for hernia development include "history of hernia or prior hernia repair (including childhood), old age, male sex", respiratory problems like chronic cough, congestion, smoking history or family history of hernia ${ }^{2}$

\section{Case Details}

Patient is a 62 year old male who reports to physician's office with a chief complaint of painless inguinal swelling bilaterally along with feeling of fullness in scrotum for the past two years which is exacerbated by standing or walking but is relieved by sitting and lying down. Patient denies any other associated complaints like fever, nausea, vomiting, loose bowel movements or urinary symptoms. Patient's past medical history is significant for Type 2
Diabetes Mellitus. Denies any drug allergies. Also denies smoking, drinking or illicit drug use.

\section{Examination}

Bilateral masses adjacent to the scrotum were palpated and were transilluminable. In addition, there was protrusion of bowel contents in the inguinal region which was confirmed when asked to cough or stand up. Patient denies any pain or tenderness on palpation in the inguinal area. Remainder of the exam was nonsignificant.

\section{Investigative Reports}

Abdominal and scrotal ultrasound: Demonstrated an encysted hydrocele bilaterally with cystic fluid filled cavities seen along with testicular tissue (Figure 1).

Complete Blood Count, Basic Metabolic Panel and Liver Function Tests: Within Normal Limits.

Fasting Blood sugar was 107; HbA1C: 5.1 within normal limits.

\section{Interventions and outcomes}

A direct inguinal hernia along with possible hydrocele was suspected bilaterally. Patient was scheduled for a modified shouldice repair. An open bilateral inguinal hernia repair with orchiopexy was performed (Figure 2).

Upon surgical exploration, left \& right scrotal swelling with encysted hydroceles of cord and epididymis were found. Hydroceles were excised (Figure 3), hernia sac was reduced and mesh Vypro 2.0 was placed over the superficial inguinal ring along with tightening of the deep inguinal ring. Urinary catheterization was performed to prevent retention. Specimens were sent for biopsy.

Correspondence: Smit Shah; 112 Montgomery Street, Apt 3G, Highland Park, NJ 08904, USA. E-mail: spshah1991@gmail.com.

South East Asia Journal of Public Health 2017;7(1):62-65. (C) 2017 Shah \& Shah, publisher and licensee Public Health Foundation Bangladesh. This is an Open Access article which permits unrestricted non-commercial use, provided the original work is properly cited. 


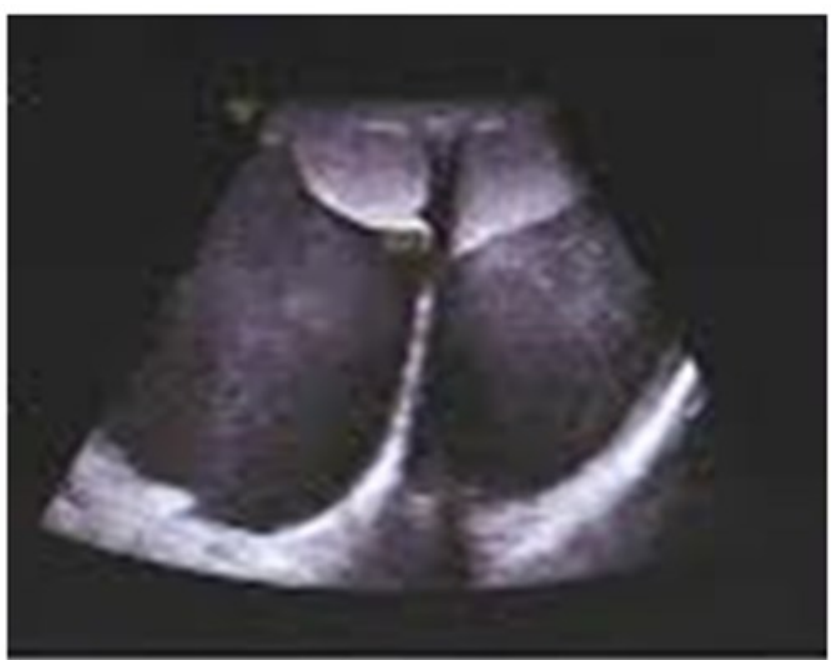

Figure 1: Encysted hydrocele visualized bilaterally on testicular ultrasound. Cystic fluid filled cavity seen along with testicular tissue. This mass was a transilluminable encysted hydrocele of spermatic cord.
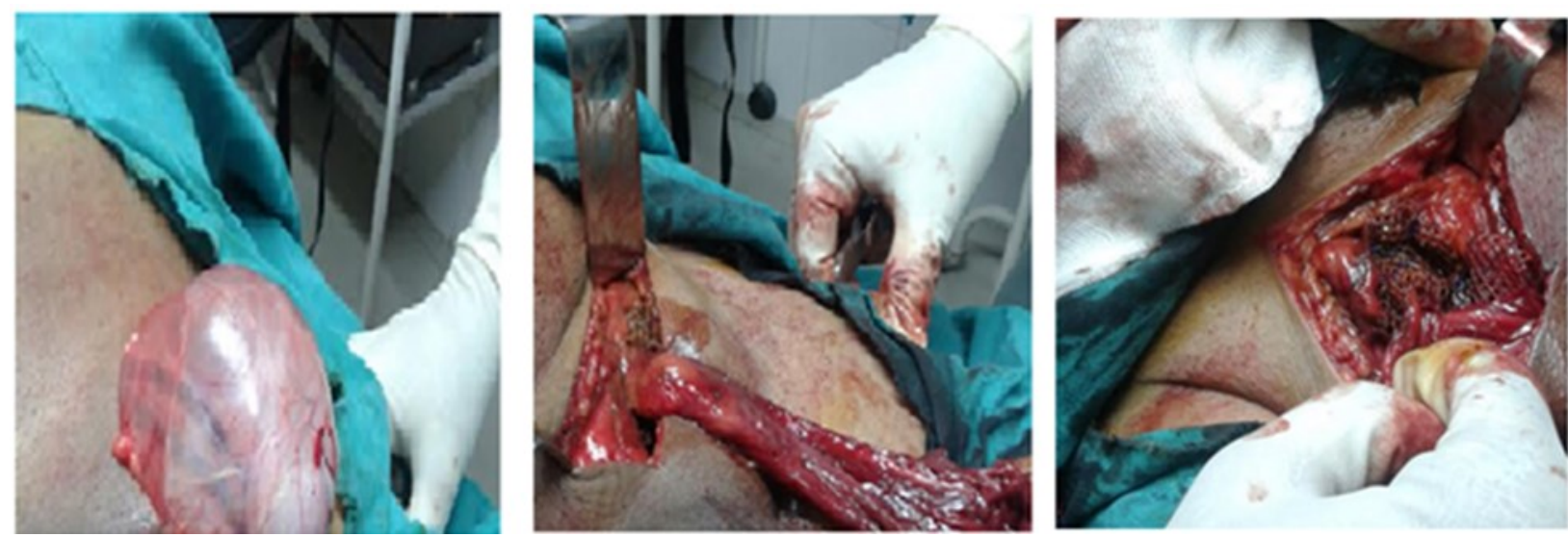

Figure 2: Hernia sac in the left inguinal region with similar presentation was seen on the right side as well (Left); Hydrocele is out of the view and will be excised later. Spermatic cord protruding from the superficial inguinal ring exposed on the left side (Middle). Placement of mesh over the superficial inguinal ring to reduce the direct hernia along with tightening of the internal inguinal ring to prevent future occurrence of indirect inguinal hernias followed by bilateral orchiopexy (Right).

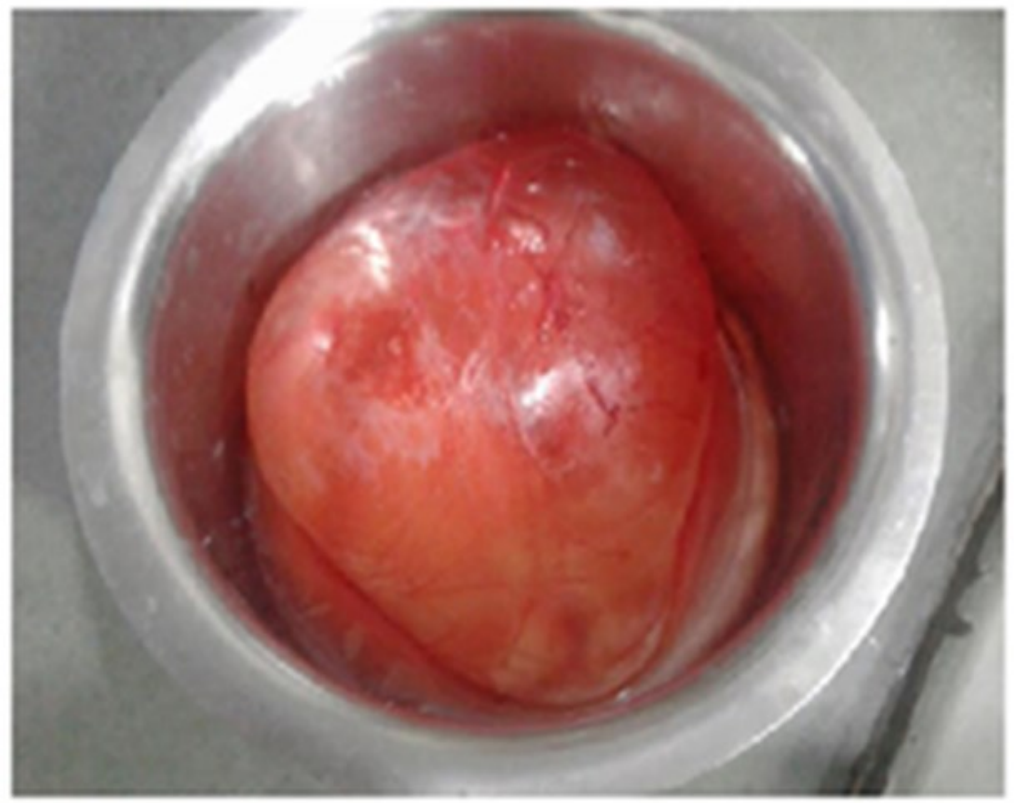

Figure 3: Excised hydrocele: well circumscribed cystic mass excised from the supero-lateral aspect of right testis. Similar mass at an identical location was excised from the left side as well 
Post-operative follow up for 6 months demonstrated clean dry surgical incision, no recurrence of hernia and relief of symptoms that are mentioned in initial chief complaint.

\section{Discussion}

Primarily, adult hydroceles are usually caused by lack of fluid reabsorption from the remnant piece of tunica vaginalis around the scrotal sac. ${ }^{3}$ In underdeveloped countries however, there are many additional etiologies which includes chronic blockage of the lymphatic system secondary to parasitic infections like $W$. Bancrofti ${ }^{4}$ Interestingly, there are also additional common presentations of hydrocele, one of which is an encysted hydrocele of the spermatic cord. There are two basic types of hydroceles which are caused either by: (1) incomplete closure from proximal to distal end of processus vaginalis during the descent of testis i.e. encysted type which is transilluminable and (2) via failure of closure of proximal processus vaginalis with an opening to the peritoneal cavity which can present with inguinal lymphadenopathy and mimic an inguinal hernia also known as funicular type. ${ }^{5}$ This demonstrates the importance of performing an inguinal ultrasound to determine the underlying etiology of any inguinal swelling.

As discussed above, modified shouldice repair with a hydrocele excision was performed. The basic steps of shouldice repair include ${ }^{6}:$ (1) isolation of the spermatic cord after dissecting the cremastric muscle in order to create the medial and lateral flaps around the spermatic cord, (2) check the femoral space by palpating the cooper's ligament in order to check for any bowel tissue which is a regular part of any inguinal surgical exploration. Final step is to reconstruct the inguinal canal including the superficial and deep inguinal rings therefore restoring the natural anatomy of the spermatic cord after hydrocele excision.

Reconstructing the inguinal rings and inguinal canal includes the following steps. Initially, medial to lateral reconstruction includes approximating the inferolateral flap of the transversalis fascia and suturing it to the lateral aspect of rectus sheath and then move laterally to the inferior oblique \& transverse abdominis muscle. This creates an internal inguinal ring and prevents the recurrence of future indirect inguinal hernia. Finally, imbrication of external and internal oblique muscles from the medial aspect of internal inguinal ring followed by reconstruction of the remaining inguinal canal from the previously excised cribiformis fascia and mobilization of the lateral flap of external oblique (Figure 4). ${ }^{6}$ Refer to Figure 1, 2 and 3 for gross description of hernia sac, spermatic cord and mesh placement.

Finally, bilateral orchiopexy was performed to ensure that spermatic cord and its contents and placed appropriately in their normal anatomical location in the scrotal sac before final surgical closure is performed. Post-operative follow up at 1 week, 1 month, 3 months and 6 months demonstrated well healed surgical wound and no recurrence of hernia. Patient denied any symptoms of inguinal swelling and feeling of fullness in scrotum.

In summary, this patient underwent a modified shouldice repair which included: hydrocele removal, the repair procedure as described above, and finally placement of a mesh on the superficial inguinal ring to prevent future recurrences of direct inguinal hernia. According to a systematic review performed by Amato et. $a l^{7}$, patients with only shouldice repair (that is usually without mesh) had a higher rate of hernia recurrence as compared to that of other techniques like Lichtenstein repair with mesh placement. ${ }^{7}$ So it was important for us to combine both traditional shouldice technique along with mesh placement to ensure a better surgical outcome.

Failure to treat hydroceles can lead to many different complications including: compression of the testes which can lead to postero-inferior displacement which can be painless initially but can cause scrotal pain \& eventual testicular atrophy secondary to pressure effect, calcification, transformation into a hematocele and infection i.e. pyelocele. ${ }^{8}$ On imaging, hydroceles are

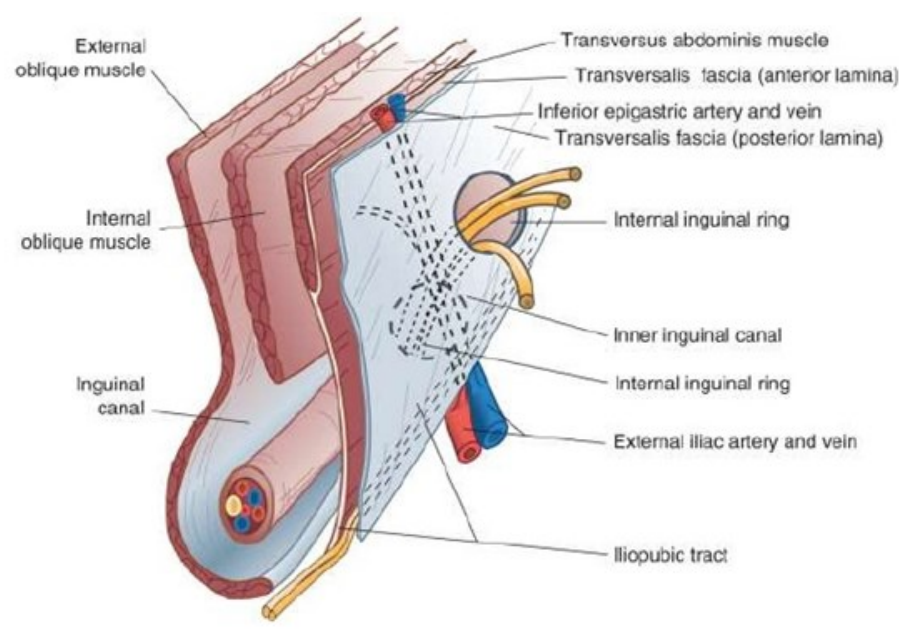

Figure 4: Anatomy of the inguinal canal and its surrounding structures ${ }^{10}$ 
characterized by a fluid collection surrounding the testis. On pathologic examination, they have a pink appearing smoother inner and outer wall along with loose fibrovascular connective tissue with thin lining. ${ }^{9}$ If it is chronic then we expect to see inflammatory changes along with calcification \& fibrous tissue.

\section{Conclusion}

We have presented a case of a patient with bilateral inguinal hernias with encysted hydrocele who underwent a modified shouldice repair which included: hydrocele excision, bilateral orchiopexy and traditional shouldice repair with mesh placement. Future research might include following patients \& determine the rate of hernia recurrence who have undergone modified shouldice technique by comparing them with patients who have undergone either only mesh repair or only shouldice repair.

\section{References}

1. Rutkow I, Robbins A. Demographic, classificatory, and socioeconomic aspects of hernia repair in the United States. Surg Clin North Am 1993;73 (3):413-26.

2. McIntosh A, Hutchinson A, Roberts A, Withers $\mathrm{H}$. Evidence-based management of groin hernia in primary care-a systematic review. Fam Pract 2000;17(5):442-7.

3. Parks K, Leung L. Recurrent Hydrocele. J Family Med and Prim Care 2013;2(1):109-10.
4. Medscape. Filarial Hydrocele. http:// emedicine.medscape.com/article/438525overview (accessed June 2017)

5. Manimaran D, Karthikeyan T, Khan D. Encysted Spermatic Cord Hydrocele in a 60-Year-Old, Mimicking Incarcerated Inguinal Hernia: A Case Report. J Clin Diagn Res 2014;8(2):153-4.

6. Chan C, Chan G. The Shouldice technique for the treatment of inguinal hernia. J Minim Access Surg 2006;2(3):124-8.

7. Amato B, Moja L, Panico S, Persico G, Rispoli $\mathrm{C}$, Rocco N, et al. Shouldice technique versus other open techniques for inguinal hernia repair. Cochrane Database Syst Rev 2009;(4):1543.

8. Medindia. Hydrocele. http://www.medindia.net/ patients/patientinfo/hydrocele-complications.htm (accessed June 2017)

9. PathologyOutlines.com. Testis and epididymis Nonneoplastic testicular lesions and Hydrocele. http://www.pathologyoutlines.com/topic/ testishydrocele.html (accessed June 2017)

10. Slideshare.net. Surgical Options in the management of Hernia Repair. https:// www.slideshare.net/safarmas/surgical-options-in -the-management-of-hernia-repair (accessed June 2017) 